\title{
GCU
}

Glasgow Caledonian

University

University for the Common Good

\section{Upper limb rehabilitation interventions using virtual reality for people with multiple sclerosis: a systematic review}

Webster, Amy; Poyade, Matthieu; Rooney, Scott; Paul, Lorna

Published in:

Multiple Sclerosis and Related Disorders

DOI:

10.1016/j.msard.2020.102610

Publication date:

2021

Document Version

Author accepted manuscript

Link to publication in ResearchOnline

Citation for published version (Harvard):

Webster, A, Poyade, M, Rooney, S \& Paul, L 2021, 'Upper limb rehabilitation interventions using virtual reality

for people with multiple sclerosis: a systematic review', Multiple Sclerosis and Related Disorders, vol. 47, 102610. https://doi.org/10.1016/j.msard.2020.102610

\section{General rights}

Copyright and moral rights for the publications made accessible in the public portal are retained by the authors and/or other copyright owners and it is a condition of accessing publications that users recognise and abide by the legal requirements associated with these rights.

Take down policy

If you believe that this document breaches copyright please view our takedown policy at https://edshare.gcu.ac.uk/id/eprint/5179 for details of how to contact us. 
Upper limb rehabilitation interventions using virtual reality for people with multiple sclerosis: a systematic review

Author names and affiliations:

Amy Webster ${ }^{\mathrm{a}}$, Dr Matthieu Poyade ${ }^{\mathrm{b}}$, Scott Rooney ${ }^{\mathrm{a}}$ and Prof Lorna Paul ${ }^{\mathrm{a}}$

${ }^{a}$ School of Health and Life Sciences, Glasgow Caledonian University.

${ }^{\mathrm{b}}$ School of Simulation and Visualisation, Glasgow School of Art.

Corresponding Author:

Amy Webster, School of Health and Life Sciences, Glasgow Caledonian University, Cowcaddens Road, Glasgow, G4 0BA. Email address: Amy.Webster@gcu.ac.uk 


\begin{abstract}
Background: Research on Virtual Reality (VR) based motor rehabilitation for people with multiple sclerosis (MS) is rapidly growing in popularity, although few studies have focused on the upper limb (UL). The aims of this review were to investigate the effect of VR interventions on UL function in people with MS and determine if the type of VR intervention influences intervention effect.

Method: Five databases (IEEE Xplore, MEDLINE, ProQuest Central (Health \& Medical Collection), Science Direct and Web of Science Core Collection) were searched using keywords that relating to MS, VR and UL.

Results: Ten articles were included, six randomised controlled trials, three cohort studies and one pilot observational study. Both commercial and custom VR technologies were used in interventions, along with combination approaches using robotics, electrical stimulation and occupational therapy. Using the Nine Hole Peg Test, two studies found significant improvements within groups, one found that VR was more effective than another gaming approach. Significant improvements in other UL measures were in the Fugl-Meyer Assessment for the proximal arm; handgrip; perceived strength; Jebsen-Taylor Hand Function Test; Wolf Motor Function Test; active range of motion and trajectory measures after VR intervention. There were conflicting results regarding if VR was more effective than conventional approaches.
\end{abstract}

Conclusion: There is therefore some evidence that VR is effective in improving motor function in the UL, however, there is no clear consensus on which VR based approaches are the most effective, or the optimum intervention duration and intensity. Moreover, as many of the studies had non-immersive approaches it is hard to determine how effective immersion based approaches maybe in such specific context.

Keywords: multiple sclerosis, virtual reality, upper limb, rehabilitation 1

\footnotetext{
${ }^{1}$ Abbreviations: ADL (Activities of daily living); BBT (Box and Blocks Test); CPT (Convention physical training); FMA (Fugl-Meyer Assessment); HMD (Head Mounted Display); JTT (Jebsen-Taylor Hand Function Test); MS (Multiple Sclerosis); PwMS (people with MS); RCT (Randomised controlled trial); Serious Games (SG); UL (Upper Limb); VR (Virtual Reality); WFMT (Wolf Motor Function Test); 9HPT (9 Hole Peg Test).
} 


\section{Introduction}

Many people with multiple sclerosis (MS) have some degree of upper limb (UL) dysfunction, such as tremor, sensory deficits, weakness and loss of dexterity in one or both hands (Kister et al., 2013; Bertoni et al., 2015). Loss of motor skills can impact the individual's quality of life, employment status and activities of daily living (ADL) (Simmons et al., 2010; Goverover et al., 2017). Dexterity problems can also result in higher healthcare related costs, due to compensatory alterations needed for home living and possible long-term care (Koch et al., 2014). This highlights the importance of finding effective treatments that target UL impairment and could potentially improve the quality of life for people with MS.

Current evidence suggests that rehabilitation can contribute to improving UL motor function in people with MS (Gandolfi et al., 2018; Gervasoni et al., 2019) however this evidence is limited due to UL rehabilitation interventions being historically understudied, despite the impact of UL impairment in people with MS (Kraft et al., 2014). One promising yet relatively new strategy of motor rehabilitation research is the use of Virtual Reality (VR). VR makes use of interactive simulations that can be built upon gamified approaches typically found in Serious Games (SG). The increased interest in VR based strategies are due to the reported advantages, such as improving motivation and enjoyment, providing real-time feedback in a safe virtual environment and having the potential to be used where the user feels comfortable, such as the home (Lange et al., 2012). Two important principles of VR are immersion and presence; immersion relates to the overall sensation of experiencing the virtual world, whereas presence is the psychological state of feeling that the user is within it (Mütterlein, 2018; Slater, 2018). There are different levels of immersion: low, semi and full (Miller and Bugnariu, 2016). Low immersion systems typically involve computer-generated environments monoscopically displayed on computer monitors (Kaplan-Rakowski and Gruber, 2019); semi immersion involves larger stereoscopic visual displays with occasional motion capture; and full immersion frequently involves the use of head mounted displays (HMDs) (Miller and Bugnariu, 2016). While full immersion is not necessary for the classification of VR; higher immersion is positively received by users as it increases motivation, lowers cognitive workload and can help improve task success (Lum et al., 2018; Yao and Kim, 2019). However, it remains unclear whether full immersion is more effective in improving motor rehabilitation outcomes when compared to lower immersion (Rose et al., 2018).

Despite the potential benefits of VR, research relating to the effectiveness of VR in MS is scarce especially regarding UL function. Previous systematic reviews conducted on VR interventions in MS have primarily focused on gait or other lower limb related outcomes (Casuso-Holgado et al., 2018), or included both upper and lower limb measures (Massetti et al., 2016; Maggio et al., 2019). Therefore, there is a need to explore the use of VR in UL rehabilitation due to the prevalence and impact of UL 
motor dysfunction in people with MS, and to provide summary of evidence for best practice for researchers and healthcare professionals. With increasing research and interest in UL in recent years, it is timely to explore the effectiveness and suitability of VR in UL function in MS.

The aim of this systematic review is to investigate the effect of VR interventions for UL rehabilitation in people with MS by exploring if: (1) VR-based rehabilitation interventions are effective in improving UL function for people with MS; (2) immersive VR based rehabilitation strategies are more effective in improving UL function compared to other conventional methods of rehabilitation.

\section{Methods:}

\subsection{Search Strategy}

Prior to conducting the review, the protocol and search strategy were registered on the PROSPERO database in March 2020 (ref CRD42020175370). Searches were performed in May 2020 of the following five databases from inception: IEEE Xplore, MEDLINE (via ProQuest), ProQuest Central (Health \& Medical Collection), Science Direct and Web of Science (Core Collection). Keywords searched were related to MS, VR and UL, and searches were kept consistent as possible between databases (Supplementary Table 1). Reference lists of the eligible articles were also searched for relevant articles and their citations checked.

\subsection{Inclusion and Exclusion Criteria}

Articles were included if they met the following criteria 1) include data on adults with MS, if people with MS were included alongside other clinical populations, the data regarding MS must be extractable; 2) report the use of a VR intervention; 3) use an outcome measure to quantify UL impairment, performance or function. For the purposes of this review, to be classified as VR, interventions must demonstrate interactivity within a virtual environment and a feeling of perceived presence by the user. There were no exclusions based on study design or the duration and frequency of interventions. Articles were excluded if they were not full texts, they were grey literature, conference abstracts, reviews or not available in English.

\subsection{Eligibility Screening}

Search results were exported to Covidence systematic review software (Veritas Health Innovation, Melbourne, Australia. Available at covidence.org). Two independent reviewers (AW \& LP) reviewed the title and abstracts for eligibility; two independent reviewers (AW \& SR) then read full text of the remaining articles against the inclusion exclusion criteria. Any disparities were resolved through consensus with a third reviewer (LP) if necessary.

\subsection{Quality Assessment}


Quality of the included articles was assessed using the Effective Public Health Practice Project (EPHPP) Quality Assessment Tool for Quantitative Studies. The EPHPP tool assesses the quality of a broad range of study design, such as randomised controlled trials (RCT), controlled trials and observational studies. It has shown good validity in the assessment of health-related studies (Thomas et al., 2004; Armijo-Olivo et al., 2012). It consists of six sections: selection bias, study design, confounders, blinding, data collection method, and withdrawals and dropouts. Each section is assigned a score of "strong” (1), "moderate" (2) or "weak" (3). The scores are then collated and a global rating of strong (1), moderate (2) or weak (3) is given for the overall article. Two reviewers (AW \& SR) independently assessed the quality of the included articles. Any disparities in the quality assessment were resolved through consensus with a third reviewer (LP) if necessary. No studies were excluded based on the results of the quality assessment.

\subsection{Data Extraction}

One reviewer (AW) extracted data into a standard data extraction table. Data were extracted in relation to: study details (authors, year of publication and study design); participant data (age, sex, disability, disease duration, MS type, controls); intervention (frequency, duration, specific virtual reality technology used, serious or commercial games), UL movements targeted (unilateral or bilateral movements), UL outcome measures, and the main findings from the study.

\subsection{Data Synthesis}

The heterogeneity of outcome measures across articles meant that a meta-analysis was not possible. Therefore, a narrative synthesis of the data was conducted, detailing the findings, the intervention protocol, the measured outcomes for each study, and the studies' strengths and weaknesses. The different VR interventions were described and, if appropriate, were compared to determine which were more effective in improving UL function.

\section{Results}

\subsection{Study Selection}

231 articles were found using the search strategy. After duplicates were removed, 167 articles were screened for eligibility and 146 articles were excluded from the title and abstract screening stage. 21 full texts were then screened for eligibility and 11 articles were excluded. Therefore, 10 were accepted for inclusion in the review. Reasons for exclusion of the 11 full text articles were: 6 articles did not use VR as an intervention; 2 articles did not present any UL measures; 2 articles did not meet the classification of VR; one article was a review paper that had no extractable data on MS patients using VR as an intervention for UL improvements (Fig. 1).

[Figure 1 Near Here] 


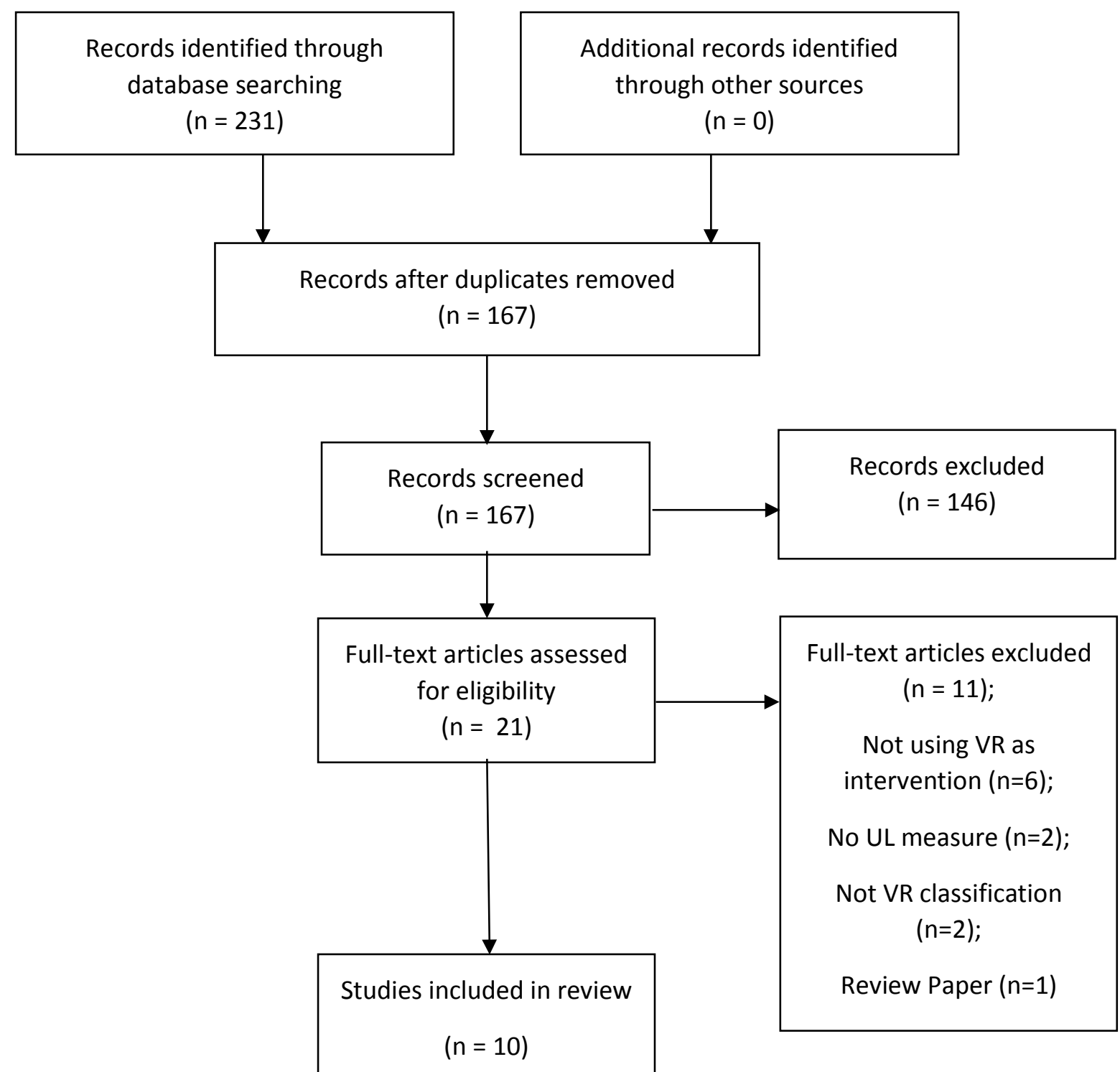

Figure 1: PRISMA (Preferred Reporting Items for Systematic Reviews and Meta-Analyses) of search results and screening. 
Table 1: Data Extraction of virtual reality-based interventions for improving upper limb studies within MS.

\section{Study and Participants \\ Design \\ (MS type, sex, age, EDSS, duration of disease) \\ Intervention \\ (VR Technology, Tasks, Duration).}

\section{UL Outcome Unilateral or Main Findings Measures \\ Bilateral \\ Intervention. \\ (Overall Conclusions).}

\section{(UL assessed)}

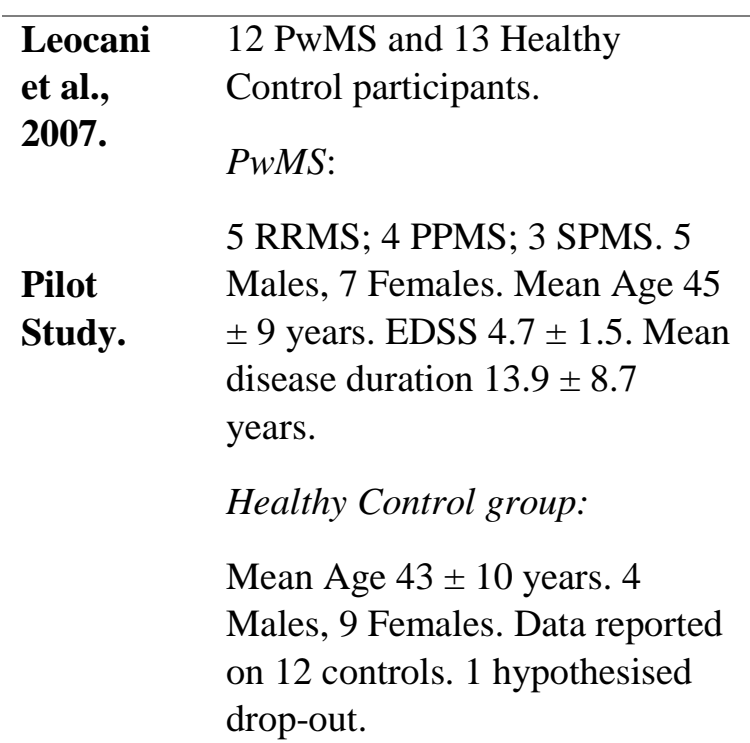

Khymeia SRL visual
display and electromagnetic
sensors.
Tracking (object on screen)
visual cue with finger
(movement of all UL).
One-day duration. 12
training sessions lasting 20
minutes. 2 minute breaks
between each session.
Same intervention for both
groups.

\begin{tabular}{|c|c|c|}
\hline $\begin{array}{l}\text { Distance } \\
\text { errors in } \\
\text { frontal }(\mathrm{x}, \mathrm{z}\end{array}$ & $\begin{array}{l}\text { Unilateral. } \\
25 \text { dominant. }\end{array}$ & $\begin{array}{l}6 \text { PwMS had over } 20 \% \text { improvement in } \\
\text { frontal plane, whereas only } 4 \text { improved } \\
\text { by } 20 \% \text { in depth plane. }\end{array}$ \\
\hline $\begin{array}{l}\text { axes) and } \\
\text { depth } \\
\text { (horizontal } x, y \text {; } \\
\text { vertical }\end{array}$ & 0 left. & $\begin{array}{l}\text { Significantly higher improvements for } \\
\text { PwMS in the frontal plane than in the } \\
\text { depth planes }(p<0.05) \text {. PwMS }\end{array}$ \\
\hline $\begin{array}{l}y, z \text { axes) } \\
\text { planes by the } \\
\text { (finger) }\end{array}$ & & $\begin{array}{l}\text { improved significantly less compared } \\
\text { with control group in the depth planes ( } p \\
<0.05) \text {. }\end{array}$ \\
\hline
\end{tabular}

trajectory.
6 PwMS had over 20\% improvement in frontal plane, whereas only 4 improved Significantly higher improvements for PwMS in the frontal plane than in the Pw $<0.05$ ).

\begin{tabular}{|c|c|}
\hline $\begin{array}{l}\text { Mahajan } \\
\text { et al., } \\
2014\end{array}$ & $\begin{array}{l}11 \text { PwMS. } 2 \text { Participant Drop } \\
\text { Out. Unspecified MS. } 5 \text { females, } \\
6 \text { males. }\end{array}$ \\
\hline RCT & $\begin{array}{l}5 \text { PwMS in isometric group. } \\
\text { Mean Age } 56.82 \pm 3.47 \text { years } \\
\text { (isometric group). }\end{array}$ \\
\hline
\end{tabular}
Virtual driving simulator
with either isometric (force
driven) or compliant
(position driven) joystick
with either standard, or an
adapted algorithm for
tremor (MSPFA) or tremor
Multiple
measures from
trajectory data
of task and
throughput
(bits per
second).
and fatigue (MSPFA FA)
Unilateral.
Data on treated
UL NR.

\author{
Mean \pm SD Throughput (bits per \\ second) Higher value means better \\ performance. \\ Isometric: \\ Standard: $0.234 \pm 0.03$
}


EDSS and disease duration NR.

Control:

6 PwMS, in compliant mode.

Mean Age $54.89 \pm 12.33$ years

(compliant mode).

EDSS and disease duration NR. to evaluate joystick

performance.

10 days apart. 6 repetitions

on each trial.
Two visits between 2 and

MSPFA: $0.255 \pm 0.01$

MSPFA_FA: $0.303 \pm 0.09$

Compliant:

Standard: $0.305 \pm 0.10$

MSPFA: $0.385 \pm 0.18$

MSPFA_FA: $0.376 \pm 0.13$

ARAT (Mean \pm SD ( $p$ value). \% change) with functional electrical $\quad$ MAM-36 and stimulation and VR display. FMA.

2 dominant; 2 non-dominant.

Baseline: $45.6 \pm 10.50$; Post: $46.2 \pm 9.55$ $(p=1.0) .1 .05 \%$.

Reaching task using virtual

environment.

2 right; 3 left.

$\underline{9 H P T}$ (Mean seconds $\pm \mathrm{SD}, p$ value, \% change)

18 one-hour training

sessions, over 10 weeks.
Baseline: $145.3 \pm 96.39$; Post: $116.3 \pm$ $87.91(p=0.465) .8 .17 \%$.

$\underline{M A M-36}$ (Mean $\pm \mathrm{SD}, p$ value, $\%$ change)

Baseline: $46.8 \pm 9.99$; Post: $50.4 \pm 8.05$ $(p=0.345)$. 3.6\%.

FMA Proximal Arm (Mean \pm SD, $p$ value, \% change)

Baseline: $22.4 \pm 4.62$; Post: $28.0 \pm 6.08$ ( $p=0.039) .15 .56 \%$.

FMA Distal Arm (Mean $\pm \mathrm{SD}, p$ value, \% change)

Baseline: $22.4 \pm 2.5$; Post: $22.8 \pm 3.7(p$ 
= 0.465). $8.17 \%$.

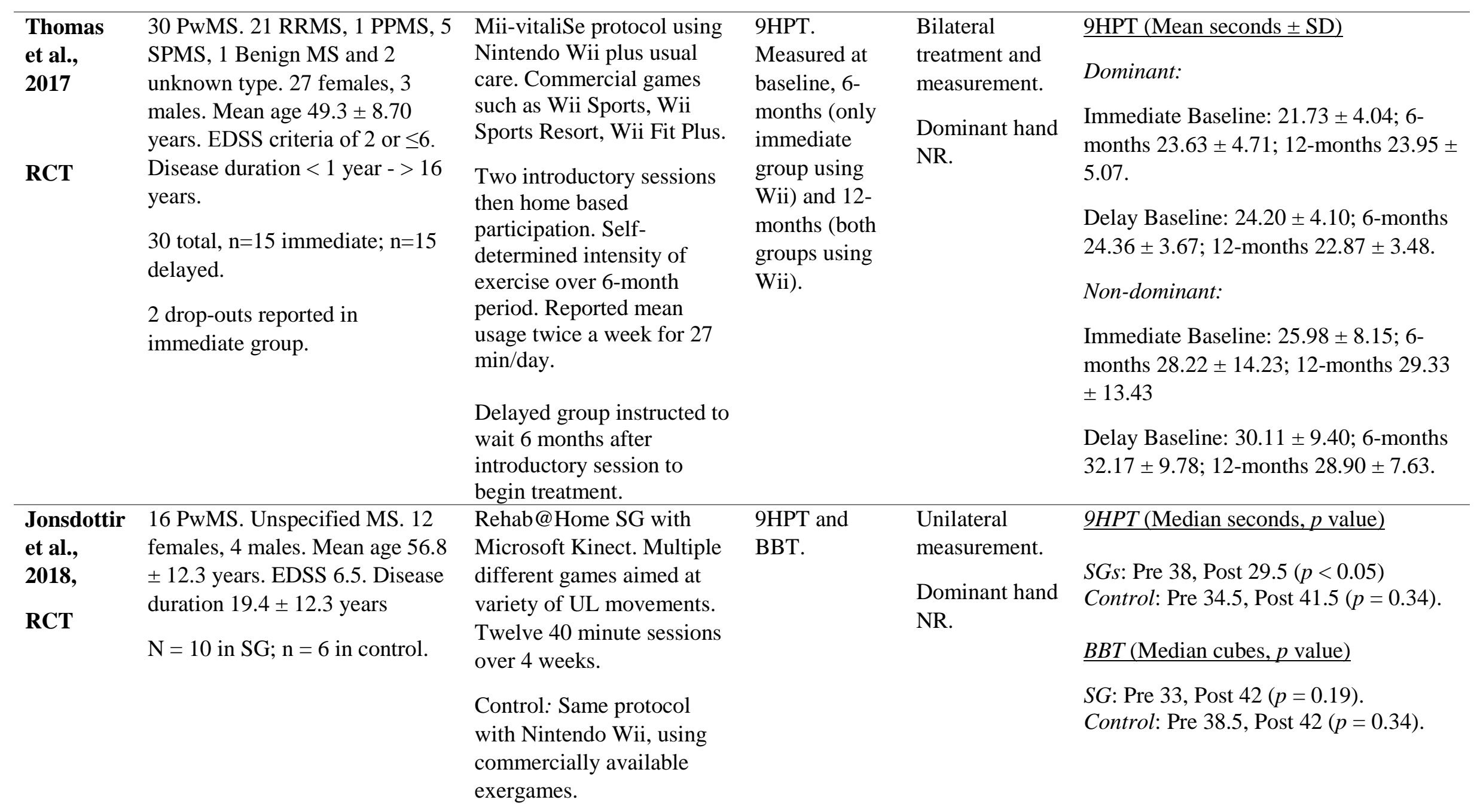




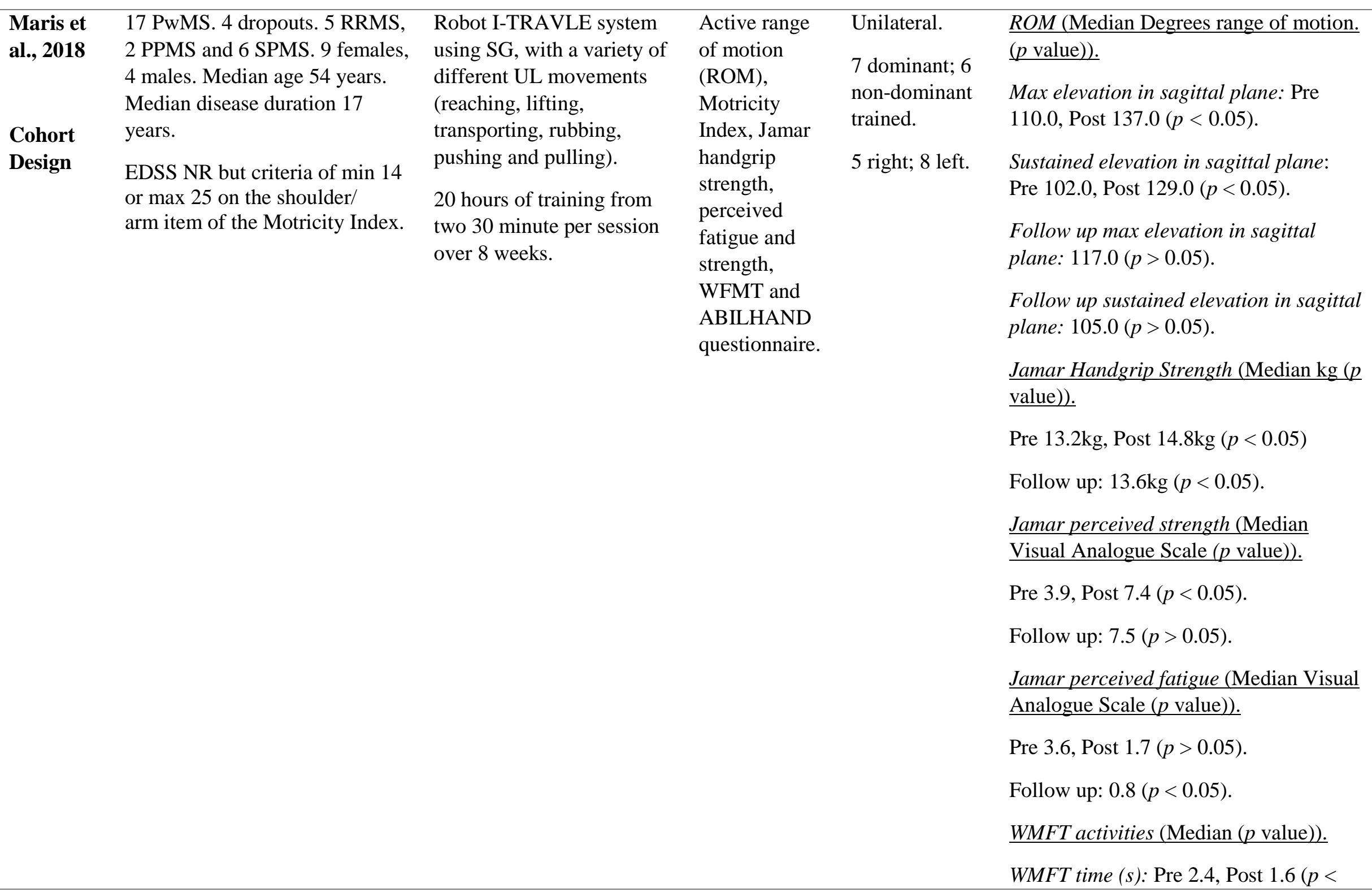


WMFT FA: Pre 4.0, Post $5.0(p<0.05)$.

Follow up WMFT time: $1.4(p<0.05)$.

Follow up WMFT FA: $5.0(p<0.05)$.

\begin{tabular}{lll}
\hline $\begin{array}{l}\text { Jonsdottir } \\
\text { et al., }\end{array}$ & $\begin{array}{l}\text { 18 PwMS. 11 RRMS, 6 SPMS } \\
\text { and 1 PPMS. 12 females, 6 }\end{array}$ & $\begin{array}{l}\text { Rehab@Home SG with } \\
\text { Microsoft Kinect. }\end{array}$ \\
$\mathbf{2 0 1 9}$ & males. Mean age 56.1 \pm 10.5 & Multiple different games \\
& years. Mean EDSS 6.5 \pm 1.9. & $\begin{array}{l}\text { aimed at variety of UL } \\
\text { movements. }\end{array}$ \\
Cohort & yean disease duration 17.6 \pm 9.7 & Twelve 45 minute sessions, \\
Design & & 3-5 times a week.
\end{tabular}

9HPT and

BBT.

Self-perceived

worse arm

treated.

Unilateral

treatment.

Bilateral

measurements.

\section{HPT (Median change in seconds, $p$}

value).

Treated arm: Pre 33.6, Post 28.0, $p<$ 0.05 .

Non-treated arm: Pre 30.2, Post 25.6, $p$ $>0.05$.

\section{$\underline{B B T}$ (Median change, $p$ value).}

Treated arm: Pre 38, Post 45, $p<0.05$.

Non-treated arm: Pre 41, Post 45, $p>$ 0.05 .

$\begin{array}{ll}\text { PPT, JTT and } & \text { Bilateral } \\ \text { GPT. } & \begin{array}{l}\text { treatment and } \\ \text { measurement. }\end{array}\end{array}$

JTT Writing non-dominant hand (Mean time \pm SD):

OT: Pre $93.25 \pm 73.68$, Post $62.92 \pm$ 42.92, $p<0.05$.

$O T+V R$ : Pre $62.92 \pm 42.92$, Post 50.68 $\pm 39.57, p>0.05$.

20 sessions of VR interventions with a 30minute duration, twice a week, along with conventional treatment protocol (OT).

OT sessions twice a week,
JTT Moving large light objects with non-dominant hand (Mean time $\pm \mathrm{SD}$ ):

OT: Pre $8.15 \pm 5.03$, Post $5.78 \pm 2.05, p$ $>0.05$. 
$O T+V R$ : Pre $6.98 \pm 3.38$, Post $8.89 \pm$ 5.71, $p<0.05$.

JTT Moving large light objects with dominant hand (Mean time $\pm \mathrm{SD}$ ):

OT: Pre $6.12 \pm 1.66$, Post $5.78 \pm 2.05, p$ $<0.05$.

$O T+V R$ : Pre $5.78 \pm 2.05$, Post $6.45 \pm$ $1.87, p<0.05$.

No significant difference in improvements between groups in all outcomes.

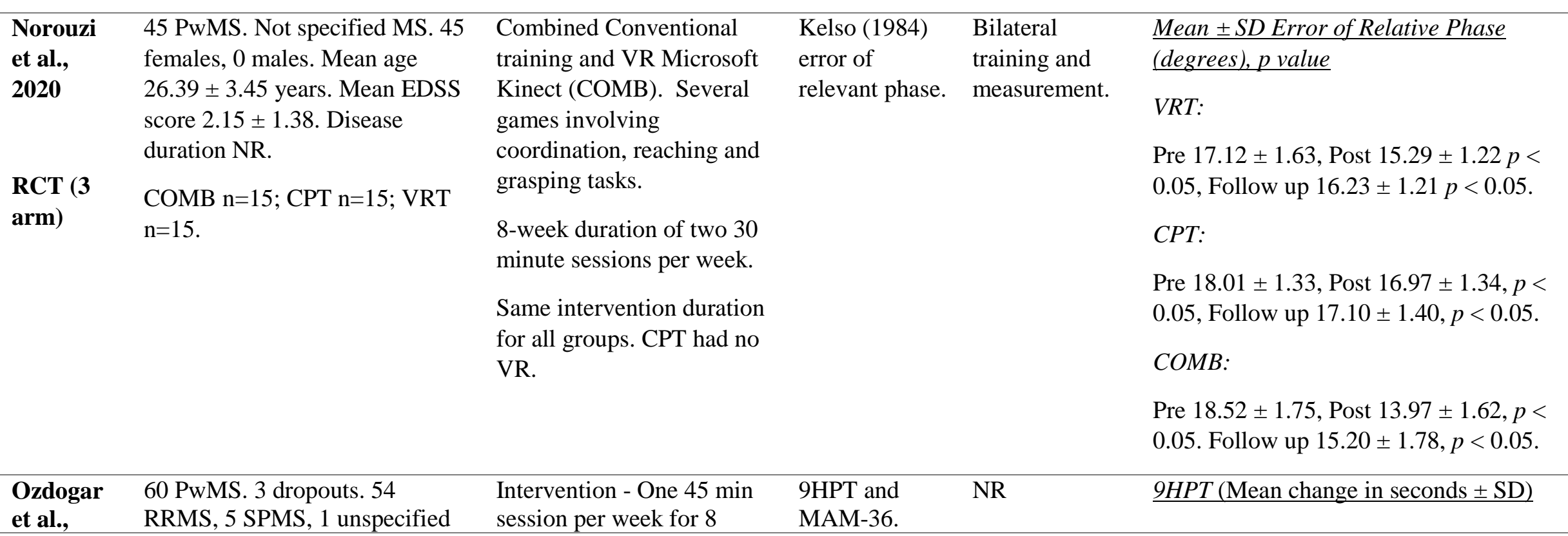




2020 MS. 43 females, 16 males and 1

arm) $\quad 2.4 \pm 1.4$. Disease duration $6.6 \pm$ 4.8 years.
RCT (3 $40.1 \pm 10.7$ years. Mean EDSS weeks.

VR: Kinect Sport Rivals using Microsoft Kinect $(n=20)$.

Conventional: Exercise based programme $(n=19)$.

Control: No therapy $(n=20)$.
$V R:-3.5 \pm 3.4, p<0.05$

Conventional: $-3.4 \pm 5.0, p<0.05$.

Control: $0.5 \pm 2.2, p>0.05$.

Group difference: 5.884, $p=0.05$.

MAM-36 (Mean score \pm SD)

$V R: 4.3 \pm 7.5, p<0.05$

Conventional: $8.5 \pm 13.4, p<0.05$.

Control: $-3.1 \pm 6.1, p<0.05$.

Group difference: 7.369, $p<0.05$.

Abbreviations: ARAT (Action Research Arm Test); BBT (Box and Blocks Test); CPT (Convention physical training); FMA (Fugl-Meyer Assessment); GPT (Grooved Pegboard Test); JTT (Jebsen-Taylor Hand Function Test); MAM-36 (The Manual Ability Measurement-36); MSPFA (Multiple Sclerosis personally fitted algorithm); MSPFA_FA (Multiple Sclerosis personally fitted algorithm with fatigue adaptation); NR (not reported); OT (Occupational therapy); PPMS (primary progressive multiple sclerosis); PPT (Purdue Pegboard Test); PwMS (People with multiple sclerosis); RCT (randomised controlled trial); RRMS (Relapse and remitting multiple sclerosis); SD (standard deviation); SG (serious games); SPMS (secondary progressive multiple sclerosis); UL (upper limb); WFMT (Wolf Motor Function Test); WMFT FA (Wolf Motor Function test functional activity); VRT (Virtual reality training); 9HPT (9 Hole Peg Test). 


\section{[Table 1 Near Here]}

\subsection{Study Design and Participants}

Six studies were RCTs (Mahajan et al., 2014; Thomas et al., 2017; Jonsdottir et al., 2018; WaliñoPaniagua et al., 2019; Norouzi et al., 2020; Ozdogar et al., 2020), three were cohort studies (Sampson et al., 2016; Maris et al., 2018; Jonsdottir et al., 2019), and one was a pilot observational study which compared the outcomes of the intervention in a MS population to a group of healthy participants (Leocani et al., 2007). Studies evaluated different joystick algorithms (Mahajan et al., 2014) or compared other VR interventions with other VR approaches (Jonsdottir et al., 2018), conventional approaches (Waliño-Paniagua et al., 2019; Norouzi et al., 2020; Ozdogar et al., 2020) and no therapy (Ozdogar et al., 2020).

All studies had relatively small sample sizes, ranging from 5 (Sampson et al., 2016) to 60 (Ozdogar et al., 2020) participants. Relapse and remitting MS (RRMS) was the most investigated type of MS, comprising $73.4 \%$ of the study population which reported MS type. RRMS was the only type of MS to be solely investigated (Waliño-Paniagua et al., 2019) and was investigated in seven of the ten articles. RRMS was studied alongside other types of MS, such as secondary progressive (Leocani et al., 2007; Sampson et al., 2016; Thomas et al., 2017; Maris et al., 2018; Jonsdottir et al., 2019; Ozdogar et al., 2020), primary progressive (Leocani et al., 2007; Sampson et al., 2016; Thomas et al., 2017; Maris et al., 2018) and begin MS (Thomas et al., 2017). There were no comparisons of outcomes across MS types in any papers, with some articles not reporting the MS type of the participants (Mahajan et al., 2014; Jonsdottir et al., 2018; Norouzi et al., 2020).

When reported, disability was measured by the Expanded Disability Status Scale (EDSS) (Kurtzke, 1983) or Motricity Index (MI). Mahajan et al. (2014), Sampson et al. (2016) and Waliño-Paniagua et al (2019) did not report any disability measure; however, they included measurable UL dysfunction as part of the inclusion criteria. Mean EDSS score ranged from $2.15 \pm 1.38$ (Norouzi et al., 2020) to 6.5 \pm 1.9 (Jonsdottir et al., 2018; Jonsdottir et al., 2019). Disease duration ranged from under 1 year (Thomas et al., 2017) to 34 years (Sampson et al., 2016). Disease duration was not reported in three articles (Mahajan et al., 2014; Waliño-Paniagua et al., 2019); Norouzi et al., 2020).

\subsection{Intervention and VR Technology}

All ten articles had different intervention protocols. The most commonly used VR technologies consisted of commercially available gaming hardware such as the Microsoft Kinect (Jonsdottir et al., 2018; Jonsdottir et al., 2019; Norouzi et al., 2020; Ozdogar et al., 2020) and the Nintendo Wii controller (Thomas et al., 2017; Jonsdottir et al., 2018). Three studies reported using the Microsoft Kinect as an interaction paradigm to track users' motion within the virtual environments of custom SG (Jonsdottir et al., 2018; Jonsdottir et al., 2019; Norouzi et al., 2020). Other VR approaches 
consisted of specifically tailored solutions that are not commercially available technology (Leocani et al., 2007; Mahajan et al., 2014; Sampson et al., 2016; Maris et al., 2018; Waliño-Paniagua et al., 2019). For instance, Leocani et al. (2007) used the Khymeia SRL visual display that works with electromagnetic input sensors, and Waliño-Paniagua et al. (2019) used a web-based video capture as motion tracking paradigms.

Other studies investigated a VR approach alongside other interventions such as robotics (Sampson et al., 2016, Maris 2018), electrical stimulation (Sampson et al., 2016) and conventional physical therapy (Waliño-Paniagua et al., 2019; Norouzi et al., 2020). Nozouri et al. (2020) assessed a VR combination method with conventional physical therapy (CPT) using two control groups (CPT alone or VR alone).

There were differences in duration and intensity of interventions investigated. The duration varied from one day (Leocani et al., 2007) to 6-month programme (Thomas et al., 2017). Sessions lasted between 20 minutes (Leocani et al., 2007) to an hour (Sampson et al, 2016), and one study did not report the intervention duration (Mahajan et al., 2014). The UL tasks were diverse across studies. Grasping and reaching were common movements in the VR tasks (Leocani et al., 2007; Jonsdottir et al., 2018; Jonsdottir et al., 2019; Norouzi et al., 2020). Jonsdottir et al. (2018 and 2019) used SG system known as Rehab@Home which comprised of four activities: organising a kitchen, moving objects, grasping flowers and following a trajectory. Norouzi et al. (2020) considered similar functional tasks to that used by Jonsdottir et al. such as grasping and moving household objects. Other tasks, such as reaching, lifting and turning, were mediated by robot-mediated therapy to provide strength and resistance training (Sampson et al., 2016; Maris et al., 2018), and a virtual driving simulator to train individuals to use a joystick with the aim of reducing fatigue from wheelchair use (Mahajan et al., 2014).

\subsection{UL Assessed and Outcome Measures}

The 9 Hole Peg Test (9HPT) was the most commonly used UL outcome reported in five studies (Sampson et al., 2016; Thomas et al., 2017; Jonsdottir et al., 2018; Jonsdottir et al., 2019; Ozdogar et al., 2020). Other reported objective UL measures for dexterity were Box and Blocks Test (BBT) (Jonsdottir et al., 2018, 2019); Purdue Pegboard Test (PPT), Jebsen-Taylor Hand Function Test (JTT) and Grooved Pegboard Test (GPT) (Waliño-Paniagua et al., 2019). Other objective UL outcome measures used were active range of motion of the shoulder joint (ROM), Motricity Index, Jamar handgrip strength, Wolf Motor Function Test (WFMT) (Maris et al., 2018); Action Research Arm Test (ARAT) and Fugl-Meyer Assessment (FMA) (Sampson et al., 2016). Patient reported outcomes used were visual analogue scales such as perceived fatigue and strength (Maris et al., 2018) and questionnaires such as The Manual Ability Measurement-36 (MAM-36) (Sampson et al., 2016; Ozdogar et al., 2020) and ALBIHAND questionnaire (Maris et al., 2018). The remaining methods of UL assessment were trajectory based data, including the trajectory path of the hand (Leocani et al., 
2007); joystick performance (Mahajan et al., 2014) and the errors of bimanual coordination using the principle of error of relevant phase (Norouzi et al., 2020).

Five studies treated and measured UL function in one arm (Leocani et al., 2007; Mahajan et al., 2014; Sampson et al., 2016; Jonsdottir et al., 2018; Maris et al., 2018). Leocani et al. (2007) treated the dominant arm, whereas the other studies treated the more impaired arm. Three studies had bilateral treatment and assessment (Thomas et al., 2017; Waliño-Paniagua et al., 2019; Norouzi et al., 2020). Jonsdottir et al. (2019) had unilateral treatment, but assessed both arms to observe if there were bilateral improvements. Ozdogar et al. (2020) did not report which UL was treated or assessed.

\subsection{Effectiveness of VR Interventions}

Of the five articles which used the 9HPT to measure UL function, three found significant improvements in the 9HPT in one arm after 4 weeks (Jonsdottir et al., 2018, Jonsdottir et al., 2019), and 8 weeks' intervention (Ozdogar et al., 2020). Significant reduction in time taken during the 9HPT ranged from 3.5s (Ozdogar et al., 2020) to 8.5s (Jonsdottir et al., 2018). In Thomas et al. (2017), only the delayed group had improvement in the 9HPT at 12-months, but this change after intervention was not statistically significant. Likewise, in Sampson et al. (2016), non-statistically significant improvements in the 9HPT were also reported.

Significant improvements in UL function were also found in studies which used other outcome measures. For example, using the JTT, Waliño-Paniagua et al, (2019) reported a significant improvement in dominant UL function for both occupational therapy (OT) alone and OT + VR groups, and significant improvements in non-dominant UL function for the OR + VR group. However, only the OT group demonstrated significant improvements in writing with the nondominant hand. The other UL outcomes in this study, GPT and PPT, did not show any significant improvements in either group.

In Sampson et al. (2016), there were reported significant improvements in the FMA proximal arm and no statistical improvements were found in the ARAT, 9PHT and MAM-36. In addition, Maris et al. (2018) reported significant improvements in multiple UL measures: maximum and sustained elevation in sagittal plane, handgrip strength, perceived strength and in the WMFT. Of the studies which included self-reported UL measures, significant improvements were found in the MAM-36 questionnaire for both VR and conventional physical training groups (Ozdogar et al., 2020) but not in the study by Sampson et al. 2016. Jonsdottir et al. (2019) investigated if a bilateral improvement was observable following single-arm treatment, and whilst there was improvement in both arms, only the treated arm showed statistically improved function in the BBT and 9HPT.

Trajectory based measures were found to significantly improve following intervention (Leocani et al., 2007; Norouzi et al., 2020), even with a short term intervention lasting one day, with 12 training 
sessions (Leocani et al., 2007). People with MS improved significantly less in the depth plane tracking compared to the frontal, and improved significantly less in the depth plane than the healthy control participants (Leocani et al., 2007). All groups in the study by Norouzi et al. (2020) improved with regards to the error of relevant phase, however the combined group using VR + CPT improved significantly more compared to the other groups (VR only and CPT only). Mahajan et al. (2014) demonstrated that an adapted algorithm for MS and fatigue resulted in better joystick performance, using throughput data, but was not significant between mode or algorithm. The studies using robotics and stimulation (Sampson et al., 2016; Maris et al., 2018) did not have a control or comparison group, therefore there is no evidence to suggest this combination was more effective in improving arm function than each intervention alone.

In order to assess the long-term impact of VR interventions, two studies included a follow up measure post-intervention to assess whether changes in UL function were maintained; however, these studies reported contrasting results. Maris et al. (2018) reported loss of the significant improvements in multiple measures after 3-month follow up, whereas improvements in the measured outcome (errors in bimanual coordination) were maintained 1 month after intervention in Norouzi et al. (2020).

\subsubsection{VR effectiveness compared to conventional therapy}

While there is evidence that VR based intervention can improve UL function in people with MS, the effectiveness of VR remains unclear when compared to other methods. Waliño-Paniagua et al. (2019) reported no significant differences between OT and VR combined with OT + VR for all UL outcome measures. In contrast, Norouzi et al. (2020) demonstrated that combined CPT + VR was more effective in improving UL function compared to CPT or VR alone, although assessment relied on trajectory measures. Ozdogar et al. (2020) demonstrated improvements in UL function following both VR and CPT, and these groups improved significantly more than the control group who received no therapy.

\subsection{Effect of VR Strategy on UL Rehabilitation}

Jonsdottir et al. (2018) was the only study to compare VR-based systems, and they reported that there was a significantly greater improvement in the 9HPT in the group receiving serious games using Microsoft Kinect than commercially available games using the Nintendo Wii. Studies involving commercially available games in conjunction with the Microsoft Kinect (Ozdogar et al., 2020) and Nintendo Wii reported positive user feedback (Thomas et al., 2017, Jonsdottir et al., 2018). No significant improvement of the UL function in 9HPT or BBT was reported by Jonsdottir et al., (2018), Even though the control group using the Nintendo Wii system seemed to have higher perceived health, as measured by EQ-5D visual analogue scale (EQ-VAS) and the SF-12, (Jonsdottir et al., 2018). 


\subsection{Quality Assessment}

Overall, according to the EPHPP, there were 3 strong (Jonsdottir et al., 2018; Jonsdottir et al., 2019; Waliño-Paniagua et al., 2019), 3 moderate (Thomas et al., 2017; Norouzi et al., 2020; Ozdogar et al., 2020) and 4 weak rated papers (Leocani et al., 2007; Mahajan et al., 2014; Sampson et al., 2017; Maris et al., 2018) (Table 2). Study design was not associated the quality of scores, for example, Mahajan et al. (2014), a RCT, received a weak rating, whereas Jonsdottir et al. (2019), a cohort design received a strong rating. Selection bias was moderate across all papers and there was a consistently strong rating for withdrawals and drop-outs, except for Waliño-Paniagua et al. (2019).

[Table 2 near here]

Table 2: Quality assessment results of articles using the EPHPP Quality Assessment Tool for Quantitative Studies.

\section{Global Component Ratings}

\begin{tabular}{|c|c|c|c|c|c|c|c|}
\hline Study & $\begin{array}{l}\text { A) } \\
\text { Selection } \\
\text { Bias }\end{array}$ & $\begin{array}{l}\text { B) } \\
\text { Study } \\
\text { Design }\end{array}$ & $\begin{array}{l}\text { C) } \\
\text { Confounders }\end{array}$ & $\begin{array}{l}\text { D) } \\
\text { Blinding }\end{array}$ & $\begin{array}{l}\text { E) Data } \\
\text { Collection } \\
\text { Method }\end{array}$ & $\begin{array}{l}\text { F) } \\
\text { Withdrawals } \\
\text { and Drop- } \\
\text { Outs }\end{array}$ & $\begin{array}{l}\text { Global } \\
\text { Score }\end{array}$ \\
\hline $\begin{array}{l}\text { Leocani } \\
\text { et al., } \\
2007\end{array}$ & 2 & 2 & 1 & 3 & 3 & 1 & 3 \\
\hline $\begin{array}{l}\text { Mahajan } \\
\text { et al., } \\
2014\end{array}$ & 2 & 1 & 3 & 2 & 3 & 1 & 3 \\
\hline $\begin{array}{l}\text { Sampson } \\
\text { et al., } \\
2016\end{array}$ & 2 & 2 & 3 & 3 & 1 & 1 & 3 \\
\hline $\begin{array}{l}\text { Thomas } \\
\text { et al., } \\
2017\end{array}$ & 2 & 1 & 1 & 3 & 1 & 1 & 2 \\
\hline $\begin{array}{l}\text { Jonsdottir } \\
\text { et al., } \\
2018\end{array}$ & 2 & 1 & 1 & 2 & 1 & 1 & 1 \\
\hline $\begin{array}{l}\text { Maris et } \\
\text { al., } 2018\end{array}$ & 2 & 2 & 3 & 3 & 1 & 1 & 3 \\
\hline $\begin{array}{l}\text { Jonsdottir } \\
\text { et al., } \\
2019\end{array}$ & 2 & 2 & 2 & 2 & 1 & 1 & 1 \\
\hline $\begin{array}{l}\text { Waliño- } \\
\text { Paniagua } \\
\text { et al., } \\
2019\end{array}$ & 2 & 1 & 1 & 2 & 1 & 2 & 1 \\
\hline
\end{tabular}




\begin{tabular}{llllllll}
\hline $\begin{array}{l}\text { Norouzi } \\
\text { et al., } \\
\mathbf{2 0 2 0}\end{array}$ & 2 & 1 & 1 & 3 & 1 & 1 & 2 \\
\hline $\begin{array}{l}\text { Ozdogar } \\
\text { et al., }\end{array}$ & 2 & 1 & 1 & 3 & 1 & 1 & 2 \\
$\mathbf{2 0 2 0}$ & & & & & & & \\
\hline
\end{tabular}

Key: 1) Strong; 2) Moderate; 3) Weak.

\section{Discussion}

Despite the limited number of studies eligible for this review, there is early evidence to suggest that VR based rehabilitation has the potential to improve UL functions within people with MS. This evidence comes from the use of valid and reliable UL measures (9HPT, BBT, JTT, FMA and WMFT) showing significant improvements in function after VR intervention. Significantly greater improvements in UL function were reported by only one study following a VR intervention compared to conventional therapy, which may reflect the fact that most if not all studies were underpowered. Therefore, the effectiveness of VR interventions in UL rehabilitation remains unclear. Furthermore, as immersion and presence for the user were not reported by the studies in this review, it is not possible to determine if immersive-based approaches were more effective. Only one study compared different VR technologies, and the VR strategy that provided higher immersion (Microsoft Kinect) was the only intervention to result in significant improvements in the 9HPT (Jonsdottir et al., 2018). The Microsoft Kinect offers more interactions within the virtual world, allowing patients to use their full body compared to the Nintendo Wii's handheld controller. The technical properties of both systems and their suitability for rehabilitation have been discussed (Tanaka et al., 2012) but there has been limited studies comparing the effectiveness of these systems in rehabilitation.

HMDs are often used alongside VR and can offer full immersion to the user (Sharples et al., 2008). To the knowledge of the authors, there are no studies for UL rehabilitation using HMDs in MS populations, to enable fully immersive experiences (Amin et al., 2016). The level of immersion is believed to be important for rehabilitation (Rose et al., 2018), as visualisation-based approaches have been reported to improve understanding and delivery of task (Bayyari and Tudoreanu, 2006) as well as better perception of movement performance (Ferreira Dos Santos et al., 2016). A few studies in MS have trialled HMDs in relation to gait and lower limb, and have produced promising evidence of improvement in motor recovery and importantly without adverse effects (Peruzzi et al., 2016; Ozkul et al., 2020).

Reporting the specific actions, exercises or games used within each study were generally poorly described in the articles included in this review, making it difficult to identify what specific intervention components are effective in promoting UL recovery. This information is important when comparing SG testing against commercially available applications. SG typically endeavour to offer a user friendly experience for a specific purpose and are more focused on improving function for those 
with varying skill (Rego et al., 2010). Although, as Jonsdottir et al. (2018) reported that exergames, fitness-based video games, could provide more motivational factors, future studies should explore the influence of different game types on clinical outcome, adherence, and acceptability.

Another finding from this review was the diversity of VR technology used across the included studies. Some VR approaches arguably had limited and restrictive interaction with the virtual environments (Leocani et al., 2007, Mahajan et al., 2014, Waliño-Paniagua et al., 2019). It is not surprising that the VR technology varied between the included studies, as there have been considerable technological changes in VR making them more commercially accessible and easily deployed within clinical research. However, the rapid technological advances in VR often mean technologies can quickly become outdated. From a research perspective, this means that experimental outcomes and development can quickly become obsolete. With this in mind, future studies should not be reliant on the specific VR technologies but rather focus on highlighting the specific beneficial factors of the technological concept and the intervention protocol itself (Lamers et al., 2016).

The intervention protocols within this review were different across all ten articles. All but two studies focussed solely on UL functions. The other protocols focused on overall physical activity (Thomas et al., 2017) and included lower limb and gait related activities (Thomas et al., 2017; Ozdogar et al., 2020). Another systematic review investigating the overview of UL rehabilitation strategies in MS (Lamers et al., 2016), found similar results in the inconsistency of protocols for UL rehabilitation. Furthermore, the long term effect of these interventions is unclear due to the lack of follow up in all but two studies, which gave conflicting results (Waliño-Paniagua et al., 2019, Norouzi et al., 2020).

Thomas et al. (2017) was the only study to investigate a home-based approach to rehabilitation which was supported with regular communication with physiotherapists. Whilst UL function did not significantly improve, there was high patient compliance and positive participant feedback. Other approaches such as the Kinect could be suitable for affordable home-based therapy whereas the robotic-based systems are a costly and often requires supervision (Van der Loos et al., 2016). From the perspectives of healthcare services and patients, there is a desire for affordable, enjoyable and effective home based interventions. Home based therapy eliminates the need for travel, which can be expensive and difficult for people with MS-related disabilities, and the dependence on healthcare professionals to operate the equipment (Taylor and Curran, 2015). Additionally, the COVID-19 pandemic, which forced temporary closure inpatient and outpatient facilities worldwide, highlights the benefits of investigating more accessible approaches such as tele-rehabilitation or other home-based approaches (Azhari and Parsa, 2020).

Other clinical conditions, such as Parkinson's disease and stroke, also have conflicting results in terms of the effectiveness of VR over other approaches, reporting that virtual reality may only be effective in improving function when used in combination with usual care (Dockx et al., 2016; Laver et al., 
2017). A similar finding was recently reported in a study investigating VR and hand tracking, for people with MS. The intervention group that used VR had significant improvements in UL outcome measures, however this was a combination approach of both VR and conventional therapy rather than VR alone (Cuesta-Gómez, et al, 2020). Despite the lack of consensus about VR effectiveness, there is often encouraging user feedback regarding this method and positive user opinion and input into rehabilitation is theorised to produce more long-term effects (Verma et al., 2012). VR is often reported to improve patient compliance compared to other methods, which was seen in the low number of drop-outs amongst most articles within this review. VR may therefore be more useful in long term conditions like MS, where prolonged rehabilitation is required. Overall, the optimum exposure to VR for UL rehabilitation in MS is unknown and needs further explored.

\subsection{Limitations}

There are a number of limitations with regards to this review. Due to the limited number of studies reporting the same outcome measure, it was not possible to perform a meta-analysis. There were different study designs, with six RCTs, and the remaining were observational studies. Varying scores for the validity and reliability for UL measures were used. Significant results came from multiple UL measures, however, some valid and reliable measures, such as the JTT, FMA and WMFT, were designed for stroke assessment and the validity or reliability of these tests has not been investigated in MS (Lamers and Feys, 2014). There was a small number of self-reported outcomes or outcomes assessing the effects on ADL, therefore it is unclear of the significance of the intervention from the patient perspective. The majority of articles scored a weak rating in the quality assessment, and many studies did not report important data regarding participant information (EDSS, disease duration, MS type) and which UL was treated and assessed. EDSS scores generally suggested low to moderate disability, with no severely disabled participants included in the studies. The majority of the reported MS type was RRMS (73.4\%), therefore there was limited evidence for the effect of VR-based interventions on progressive MS. Finally, the significant results from the two Jonsdottir et al. (2018; 2019) papers should be interpreted with caution, as these findings were likely derived from the same study, due to similarities between participant data and intervention design.

\section{Conclusion}

This review provides some evidence that VR may improve UL recovery in people with MS. However, it is unknown whether the VR technology used or level of immersion influences clinical outcomes. There was some evidence that VR-based approaches improved UL functionality such as dexterity, mainly demonstrated by the 9HPT data, and gross general movements, demonstrated by FMA and trajectory errors, however the results rarely reached statistical significance. The number of recent studies suggests this is a growing area of research, but there is more to learn with regards to which approach is the most effective, and if these improvements can be sustained for prolonged periods of 
time. It is unclear if the immersion that can be provided through VR would be have an impact on UL impairment and function, and the optimum protocol for integrating VR as part of a rehabilitation regime.

Declarations of Interest: None.

Acknowledgements: The authors wish to give thanks for the funding this systematic review received as part of a grant award from of the Multiple Sclerosis Society, UK.

Funding: This systematic review is funded as part of a grant award from the Multiple Sclerosis (MS) Society, UK. Multiple Sclerosis Society is a registered charity in England \& Wales number 1139257 and in Scotland registered charity number SC041990.

\section{References}

Amin, A., Gromala, D., Tong, X., Shaw, C., 2016. Immersion in cardboard VR compared to a traditional head-mounted display. Presented at the International Conference on Virtual, Augmented and Mixed Reality, Springer, pp. 269-276. https://doi.org/10.1007/978-3-319-39907-2_25

Armijo-Olivo, S., Stiles, C.R., Hagen, N.A., Biondo, P.D., Cummings, G.G., 2012. Assessment of study quality for systematic reviews: a comparison of the Cochrane Collaboration Risk of Bias Tool and the Effective Public Health Practice Project Quality Assessment Tool: methodological research. Journal of evaluation in clinical practice 18, 12-18. https://doi.org/10.1111/j.1365-2753.2010.01516.x

Azhari, A., Parsa, A., 2020. Covid-19 Outbreak Highlights: Importance of Home-Based Rehabilitation in Orthopedic Surgery. Archives of bone and joint surgery 8, 317-318. https://doi.org/10.22038/abjs.2020.47777.2350

Bayyari, A., Tudoreanu, M.E., 2006. The impact of immersive virtual reality displays on the understanding of data visualization. Presented at the Proceedings of the ACM symposium on Virtual reality software and technology, pp. 368-371. https://doi.org/10.1145/1180495.1180570

Bertoni, R., Lamers, I., Chen, C.C., Feys, P., Cattaneo, D., 2015. Unilateral and bilateral upper limb dysfunction at body functions, activity and participation levels in people with multiple sclerosis. Multiple sclerosis 21, 1566-1574. https://doi.org/10.1177/1352458514567553

Casuso-Holgado, M.J., Martín-Valero, R., Carazo, A.F., Medrano-Sánchez, E.M., Cortés-Vega, M.D., Montero-Bancalero, F.J., 2018. Effectiveness of virtual reality training for balance and gait rehabilitation in people with multiple sclerosis: a systematic review and meta-analysis. Clinical rehabilitation 32, 1220-1234. https://doi.org/10.1177/0269215518768084

Cuesta-Gómez, A., Baeza, P.S.H., Oña-Simbaña, E.D., Martínez-Medina, A., Ortiz-Comino, C., de Quirós, C.B.B., Jardón-Huete, A. and Cano-de la Cuerda, R., 2020. Effects of Virtual Reality Associated With Serious Games for Upper Limb Rehabilitation in Patients With Multiple Sclerosis. a Randomized Controlled Trial. J NeuroEngineering Rehabil 17. https://doi.org/10.1186/s12984-02000718-X

Dockx, K., Bekkers, E.M., Van den Bergh, V., Ginis, P., Rochester, L., Hausdorff, J.M., Mirelman, A., Nieuwboer, A., 2016. Virtual reality for rehabilitation in Parkinson's disease. Cochrane library 2016, CD010760. https://doi.org/10.1002/14651858.cd010760.pub2 
Ferreira Dos Santos, L., Christ, O., Mate, K., Schmidt, H., Krüger, J., Dohle, C., 2016. Movement visualisation in virtual reality rehabilitation of the lower limb: a systematic review. Biomedical engineering online 15, 144. https://doi.org/10.1186/s12938-016-0289-4

Gandolfi, M., Valè, N., Dimitrova, E.K., Mazzoleni, S., Battini, E., Benedetti, M.D., Gajofatto, A., Ferraro, F., Castelli, M., Camin, M., Filippetti, M., De Paoli, C., Chemello, E., Picelli, A., Corradi, J., Waldner, A., Saltuari, L., Smania, N., 2018. Effects of High-intensity Robot-assisted Hand Training on Upper Limb Recovery and Muscle Activity in Individuals With Multiple Sclerosis: A

Randomized, Controlled, Single-Blinded Trial. Frontiers in neurology 9, 905. https://doi.org/10.3389/fneur.2018.00905

Gervasoni, E., Cattaneo, D., Bertoni, R., Grosso, C., Bisio, A., Rovaris, M., Bove, M., 2019. Effect of arm cycling and task-oriented exercises on fatigue and upper limb performance in multiple sclerosis: a randomized crossover study. International Journal of Rehabilitation Research 42, 300-308. https://doi.org/10.1097/MRR.0000000000000362

Goverover, Y., Genova, H., Deluca, J., Chiaravalloti, N., 2017. Impact of Multiple Sclerosis on Daily Life. https://doi.org/10.1007/978-0-387-98188-8_7

Jonsdottir, J., Bertoni, R., Lawo, M., Montesano, A., Bowman, T., Gabrielli, S., 2018. Serious games for arm rehabilitation of persons with multiple sclerosis. A randomized controlled pilot study. Multiple Sclerosis and Related Disorders 19, 25-29. https://doi.org/10.1016/j.msard.2017.10.010

Jonsdottir, J., Perini, G., Ascolese, A., Bowman, T., Montesano, A., Lawo, M., Bertoni, R., 2019. Unilateral arm rehabilitation for persons with multiple sclerosis using serious games in a virtual reality approach: Bilateral treatment effect? Multiple sclerosis and related disorders 35, 76-82. https://doi.org/10.1016/j.msard.2019.07.010

Kaplan-Rakowski, R. and Gruber, A., 2019. Low-immersion versus high-immersion virtual reality: Definitions, classification, and Examples with a foreign language focus. In Proceedings of the 12th International Conference Innovation in Language Learning, 552-555.

Kister, I., Bacon, T.E., Chamot, E., Salter, A.R., Cutter, G.R., Kalina, J.T., Herbert, J., 2013. Natural History of Multiple Sclerosis Symptoms. International journal of MS care 15, 146-156. https://doi.org/10.7224/1537-2073.2012-053

Koch, M.W., Murray, T.J., Fisk, J., Greenfield, J., Bhan, V., Jacobs, P., Brown, M., Metz, L.M., 2014. Hand dexterity and direct disease related cost in multiple sclerosis. Journal of the Neurological Sciences 341, 51-54. https://doi.org/10.1016/j.jns.2014.03.047

Kraft, G.H., Amtmann, D., Bennett, S.E., Finlayson, M., Sutliff, M.H., Tullman, M., Sidovar, M., Rabinowicz, A.L., 2014. Assessment of Upper Extremity Function in Multiple Sclerosis: Review and Opinion. Postgraduate medicine 126, 102-108. https://doi.org/10.3810/pgm.2014.09.2803

Kurtzke, J.F., 1983. Rating neurologic impairment in multiple sclerosis: An expanded disability status scale (EDSS). Neurology 33, 1444. https://doi.org/10.1212/wnl.33.11.1444

Lamers, I. and Feys, P., 2014. Assessing upper limb function in multiple sclerosis. Multiple sclerosis journal, 20(7), pp.775-784. https://doi.org/10.1177/1352458514525677

Lamers, I., Maris, A., Severijns, D., Dielkens, W., Geurts, S., Van Wijmeersch, B., Feys, P., 2016. Upper Limb Rehabilitation in People With Multiple Sclerosis. Neurorehabilitation and Neural Repair 30, 773-793. https://doi.org/10.1177/1545968315624785 
Lange, B., Koenig, S., Chang, C.-Y., McConnell, E., Suma, E., Bolas, M., Rizzo, A., 2012. Designing informed game-based rehabilitation tasks leveraging advances in virtual reality. Disability and Rehabilitation 34, 1863-1870. https://doi.org/10.3109/09638288.2012.670029

Laver, K.E., Lange, B., George, S., Deutsch, J.E., Saposnik, G. and Crotty, M., 2017. Virtual reality for stroke rehabilitation. Cochrane database of systematic reviews, 11.

https://doi.org/10.1002/14651858.CD008349.pub4

Leocani, L., Comi, E., Annovazzi, P., Rovaris, M., Rossi, P., Cursi, M., Comola, M., Martinelli, V., Comi, G., 2007. Impaired Short-term Motor Learning in Multiple Sclerosis: Evidence From Virtual Reality. Neurorehabilitation and Neural Repair 21, 273-278.

https://doi.org/10.1177/1545968306294913

Lum, H.C., Greatbatch, R., Waldfogle, G., Benedict, J., 2018. How immersion, presence, emotion, \& workload differ in virtual reality and traditional game mediums. Presented at the Proceedings of the Human Factors and Ergonomics Society Annual Meeting, SAGE Publications Sage CA: Los Angeles, CA, 1474-1478. https://doi.org/10.1177/1541931218621334

Maggio, M.G., Russo, M., Cuzzola, M.F., Destro, M., La Rosa, G., Molonia, F., Bramanti, P., Lombardo, G., De Luca, R., Calabrò, R.S., 2019. Virtual reality in multiple sclerosis rehabilitation: A review on cognitive and motor outcomes. Journal of clinical neuroscience 65, 106-111.

https://doi.org/10.1016/j.jocn.2019.03.017

Mahajan, H.P., Spaeth, D.M., Dicianno, B.E., Brown, K., Cooper, R.A., 2014. Preliminary evaluation of variable compliance joystick for people with multiple sclerosis. Journal of rehabilitation research and development 51, 951-962. https://doi.org/10.1682/JRRD.2013.01.0023

Maris, A., Coninx, K., Seelen, H., Truyens, V., De Weyer, T., Geers, R., Lemmens, M., Coolen, J., Stupar, S., Lamers, I., Feys, P., 2018. The impact of robot-mediated adaptive I-TRAVLE training on impaired upper limb function in chronic stroke and multiple sclerosis.

https://doi.org/10.1080/17483107.2016.1278467

Massetti, T., Trevizan, I.L., Arab, C., Favero, F.M., Ribeiro-Papa, D.C., de Mello Monteiro, C.B., 2016. Virtual reality in multiple sclerosis - A systematic review. Multiple Sclerosis and Related Disorders 8, 107-112. https://doi.org/10.1016/j.msard.2016.05.014

Miller, H.L. and Bugnariu, N.L., 2016. Level of immersion in virtual environments impacts the ability to assess and teach social skills in autism spectrum disorder. Cyberpsychology, Behavior, and Social Networking, 19, 246-256. https://doi.org/10.1089/cyber.2014.0682

Mütterlein, J., 2018. The three pillars of virtual reality? Investigating the roles of immersion, presence, and interactivity. Presented at the Proceedings of the 51st Hawaii international conference on system sciences. https://doi.org/10.24251/HICSS.2018.174

Norouzi, E., Gerber, M., Pühse, U., Vaezmosavi, M., Brand, S., 2020. Combined virtual reality and physical training improved the bimanual coordination of women with multiple sclerosis.

Neuropsychological rehabilitation, 1-18. https://doi.org/10.1080/09602011.2020.1715231

Ozdogar, A.T., Ertekin, O., Kahraman, T., Yigit, P., Ozakbas, S., 2020. Effect of video-based exergaming on arm and cognitive function in persons with multiple sclerosis: A randomized controlled trial. Multiple Sclerosis and Related Disorders 40, 101966.

https://doi.org/10.1016/j.msard.2020.101966

Ozkul, C., Guclu-Gunduz, A., Yazici, G., Atalay Guzel, N., Irkec, C., 2020. Effect of immersive virtual reality on balance, mobility, and fatigue in patients with multiple sclerosis: A single-blinded 
randomized controlled trial. European journal of integrative medicine 35, 101092. https://doi.org/10.1016/j.eujim.2020.101092

Peruzzi, A., Cereatti, A., Della Croce, U., Mirelman, A., 2016. Effects of a virtual reality and treadmill training on gait of subjects with multiple sclerosis: a pilot study. Multiple Sclerosis and Related Disorders 5, 91-96. https://doi.org/10.1016/j.msard.2015.11.002

Rego, P., Moreira, P.M., Reis, L.P., 2010. Serious games for rehabilitation: A survey and a classification towards a taxonomy, in: 5th Iberian Conference on Information Systems and Technologies. IEEE, 1-6.

Rose, T., Nam, C.S., Chen, K.B., 2018. Immersion of virtual reality for rehabilitation - Review. Applied ergonomics 69, 153-161. https://doi.org/10.1016/j.apergo.2018.01.009

Sampson, P., Freeman, C., Coote, S., Demain, S., Feys, P., Meadmore, K., Hughes, A.-M., 2016. Using Functional Electrical Stimulation Mediated by Iterative Learning Control and Robotics to Improve Arm Movement for People With Multiple Sclerosis. IEEE Transactions on Neural Systems and Rehabilitation Engineering 24, 235-248. https://doi.org/10.1109/TNSRE.2015.2413906

Sharples, S., Cobb, S., Moody, A., Wilson, J.R., 2008. Virtual reality induced symptoms and effects (VRISE): Comparison of head mounted display (HMD), desktop and projection display systems. Displays 29, 58-69. https://doi.org/10.1016/j.displa.2007.09.005

Simmons, R.D., Tribe, K.L., McDonald, E.A., 2010. Living with multiple sclerosis: longitudinal changes in employment and the importance of symptom management. Journal of neurology 257, 926936. https://doi.org/10.1007/s00415-009-5441-7

Slater, M., 2018. Immersion and the illusion of presence in virtual reality. British Journal of Psychology 109, 431-433. https://doi.org/10.1111/bjop.12305

Tanaka, K., Parker, J.R., Baradoy, G., Sheehan, D., Holash, J.R., Katz, L., 2012. A comparison of exergaming interfaces for use in rehabilitation programs and research. Loading... 6.

Taylor, J., Curran, K., 2015. Glove-Based Technology in Hand Rehabilitation. International Journal of Innovation in the Digital Economy (IJIDE) 6, 29-49. https://doi.org/10.4018/ijide.2015010103

Thomas, B.H., Ciliska, D., Dobbins, M., Micucci, S., 2004. A process for systematically reviewing the literature: providing the research evidence for public health nursing interventions. Worldviews on Evidence-Based Nursing 1, 176-184. https://doi.org/10.1111/j.1524-475X.2004.04006.x

Thomas, S., Fazakarley, L., Thomas, P.W., Collyer, S., Brenton, S., Perring, S., Scott, R., Thomas, F., Thomas, C., Jones, K., Hickson, J., Hillier, C., 2017. Mii-vitaliSe: a pilot randomised controlled trial of a home gaming system (Nintendo Wii) to increase activity levels, vitality and well-being in people with multiple sclerosis. BMJ Open 7, e016966. https://doi.org/10.1136/bmjopen-2017-016966

Van der Loos, H.F.M., Reinkensmeyer, D.J., Guglielmelli, E., 2016. Rehabilitation and Health Care Robotics. Springer International Publishing. https://doi.org/10.1007/978-3-319-32552-1_64

Verma, R., Elg, M., Engström, J., Witell, L., Poksinska, B., 2012. Co-creation and learning in health-care service development. Journal of Service M anagement.

Waliño-Paniagua, C.N., Gómez-Calero, C., Jiménez-Trujillo, M.I., Aguirre-Tejedor, L., BermejoFranco, A., Ortiz-Gutiérrez, R.M., Cano-de-la-Cuerda, R., 2019. Effects of a Game-Based Virtual Reality Video Capture Training Program Plus Occupational Therapy on Manual Dexterity in Patients with Multiple Sclerosis: A Randomized Controlled Trial. Journal of healthcare engineering 2019, 1-7. https://doi.org/10.1155/2019/9780587 
Yao, S., Kim, G., 2019. The Effects of Immersion in a Virtual Reality Game: Presence and Physical Activity. Presented at the International Conference on Human-Computer Interaction, Springer, 234242. https://doi.org/10.1007/978-3-030-22602-2_18

\section{Supplement Table 1: Search Strategy}

\begin{tabular}{|l|l|}
\hline Database & Keywords \\
\hline IEEE Xplore, MEDLINE (via ProQuest), & (“multiple sclerosis” OR “MS” OR “PwMS”) \\
$\begin{array}{l}\text { ProQuest Central (Health \& Medical Collection) } \\
\text { and Web of Science (Core Collection) }\end{array}$ & AND (“virtual reality” OR “virtual interaction” \\
& OR “virtual environment” OR “virtual world” \\
& OR “virtual presence”) AND (“upper limb” OR \\
& arm OR shoulder OR forearm OR elbow OR \\
& wrist OR hand OR finger). \\
\hline Science Direct & (“multiple sclerosis”) AND (“virtual reality”) \\
& $\begin{array}{l}\text { AND (“upper limb” OR arm OR shoulder OR } \\
\text { forearm OR elbow OR wrist OR hand OR } \\
\text { finger). }\end{array}$ \\
\hline
\end{tabular}

